\title{
Some fractional derivatives of $A$-function of multivariable
}

\author{
R. SHARMA, B. TRIPATHI AND A. DUBEY
}

\begin{abstract}
In the present paper, we study and develop Fractional derivatives of multivariable $A$ - function. We derive two theorems which will act as the key formulas from which can obtain their special cases.

Mathematics Subject Classification 2010: 26A33, 30C45, 11B65

Keywords: Generalized multivariable A - function, Hypergeometric function, Mellin - Barnes contour integral and Horn's function.
\end{abstract}

\section{INTRODUCTION}

A number of earlier works on the subject of fractional calculus give interesting account of the theory and application of fractional calculus operators in many different areas of mathematical analysis. In this paper, we define the Fractional Derivatives involving $\mathrm{A}$ - function of multivariable and derive two main theorems involving Fractional Derivative of the product of A - function of multivariable and the Horn's function. Some new and known results are also established as special cases of our main results. The Fractional Derivative of the product of the multivariable A function and Horn's function has not been established so far, and some new Fractional Derivative formulae for the product of the multivariable A-function and Horn's function are derived by making use of generalized Leibnitz rule. Recently, Berndt and Bowman [1], Chaurasia and Godika [2], Saxena [3], Tripathi et al [4] gives some integrals and series.

Gautam and Asgar [5, 6], Ram and Kumar [7], Srivastava and Panda [8] and several other authors have evaluated some definite and indefinite integrals involving the $A$ - function of one, two and multivariables. 


\section{DEFINITION OF FRACTIONAL DERIVATIVE}

Following Oldham and Spainer [9], we define the (Riemann Liouville) fractional derivatives of a function $\mathrm{f}(\mathrm{x})$ of complex order $\vartheta$ or alternatively $(a-\vartheta)^{\text {th }}$ by the following

$$
\alpha D_{x}^{\vartheta}\{f(x)\}=\left\{\begin{array}{c}
\frac{1}{\Gamma(-\vartheta)} \int_{\alpha}^{x}(x-t)^{-\vartheta-1} f(y) d y, \operatorname{Re}(\vartheta)<0, \\
\frac{d^{n}}{d x^{n}} \alpha D_{x}^{\vartheta-k}\{f(x)\}, 0 \leq \operatorname{Re}(\vartheta)<n,
\end{array}\right.
$$

where $\mathrm{n}$ is a positive integer .

For simplicity, the special case of the Fractional Derivative Operator $\alpha D_{x}^{\vartheta}$ when $\alpha=$ 0 will be written as $\alpha D_{x}^{\vartheta}$. Thus, we have

$$
0 D_{x}^{\vartheta}=D_{x}^{\vartheta}
$$

\section{MAIN RESULTS}

THEOREM 1. If $\min \left\{\rho_{r}, \sigma_{r}\right\}>0,|\arg (x / \xi)|<\pi, \operatorname{Re}(\mathrm{m})+\rho_{r} \min \left\{\operatorname{Re}\left(b_{j}, \beta_{j}\right)\right\}>$ $-1(j=1, \ldots, r),\left|z_{r} x^{\rho_{r}}\right|<r_{1},\left|(x+\xi)^{\sigma_{r}} z_{r}\right|<r_{2}, r_{1}+r_{2}=1$; then

$$
\begin{aligned}
& D_{x}^{\vartheta}\left\{x^{m}(x+\right. \\
& \left.\xi)^{\lambda} A_{p_{r}, q_{r: Y}}^{0 . n_{r}: X}\left[\begin{array}{cr}
z_{1} x^{\rho_{1}}(x+\xi)^{\sigma_{1}} \\
\vdots & \mid, \ldots, \ldots \\
z_{r} x^{\rho_{r}}(x+\xi)^{\sigma_{r}} & , \ldots, \ldots,
\end{array}\right] G_{1}\left(\gamma, \delta, \delta^{\prime}: Z_{2} x^{\rho_{2}},(\mathrm{x}+\xi)^{\sigma_{2}} z_{3}, \ldots, \ldots, z_{r}\right)\right\} \\
& =\sum_{r, s=0}^{\infty} \frac{(\gamma)_{r+s}(\delta)_{s-r}(\delta)_{r-s}^{\prime}}{(r) !(s) !}\left(z_{2} x^{\rho_{2}}\right)^{r}\left(z_{3} \xi^{\sigma_{2}}\right)^{s} z_{r} \xi^{\lambda} x^{m-\vartheta} \sum_{R=0}^{\infty} \frac{(x / \xi)^{R}}{(R) !} \\
& A_{p_{r+2}, q_{r+2: Y}}^{0 . n_{r}+2 ; X}\left[\begin{array}{c|c}
z_{1} \xi^{\sigma_{1}} x^{\rho_{1}} & \\
\vdots & \left(-\lambda-\sigma_{2} s, \sigma_{1}, \ldots ., \sigma_{r}\right),\left(-R-m-\rho_{2} r, \rho_{1}, \ldots, \rho_{r}\right), \ldots, \ldots \ldots \\
z_{r} \xi \sigma_{r} \rho_{r} & \ldots, \ldots . .\left(R-\lambda-\sigma_{2} s, \sigma_{1}, \ldots, . . \sigma_{r}\right)\left(\vartheta-m-R-\sigma_{2} s, \rho_{1}, \ldots, \ldots, \rho_{r}\right)
\end{array}\right] .
\end{aligned}
$$

PROOF. We first replace the A - function of multivariable occurring on the left -hand - side by its Mellin -Barnes type contour integral and Horn's function $G_{1}$, and changing the order of integration and differentiation, which is readily justified in view of conditions stated above and collecting the powers of $\mathrm{x}$ and $(\mathrm{x}+\xi)$, we get

$$
\begin{aligned}
& \sum_{r, s=0}^{\infty} \frac{(\gamma)_{r+s}(\delta)_{s-r}(\delta)^{\prime}{ }_{r-s}}{(r) !(s) !} z_{2}{ }^{r} z_{3}{ }^{s} \frac{1}{(2 \pi \omega)^{r}} \int_{L_{1}}, \ldots, \int_{L_{r}} \emptyset\left(s_{1}, \ldots, s_{r}\right) \prod_{i=1}^{r} \emptyset_{i}\left(s_{i}\right) z_{i}{ }^{s_{i}} \\
& \left.\quad\left\{D_{x}^{v} x^{m+\rho_{1} s+\rho_{2} r} \alpha(x+\xi)^{\lambda+\sigma_{1} s+\sigma_{2} r}\right)\right\} \mathrm{d} s_{1}, \ldots, d s_{r}
\end{aligned}
$$


Now, applying well known binomial expansion, we have

$$
\begin{aligned}
& \sum_{r, s=0}^{\infty} \frac{(\gamma)_{r+s}(\delta)_{s-r}(\delta)^{\prime}{ }_{r-s}}{(r) !(s) !} z_{2}{ }^{r} z_{3}{ }^{s} \frac{1}{(2 \pi \omega)^{r}} \int_{L_{1}}, \ldots, \int_{L_{r}} \phi\left(s_{1}, \ldots ., s_{r}\right) \prod_{i=1}^{r} \emptyset_{i}\left(s_{i}\right) z_{i} s_{i} \xi^{\lambda+\sigma_{1} s+\sigma_{2 r}} \\
& D_{x}^{v} x^{m+\rho_{1} s+\rho_{2} r} \sum_{R=0}^{\infty}\left(\begin{array}{c}
\lambda+\sigma_{1} s+\sigma_{2} r \\
R
\end{array}\right)\left(\frac{x}{\xi}\right)^{R} \mathrm{~d} s_{1}, \ldots, d s_{r} .
\end{aligned}
$$

Making use of the formula [the result Oldham and Spanier [9]], we get

$$
\begin{aligned}
& \sum_{r, s=0}^{\infty} \frac{(\gamma)_{r+s}(\delta)_{s-r}(\delta)^{\prime}{ }_{r-s}}{(r) !(s) !} \frac{1}{(2 \pi \omega)^{r}} \int_{L_{1}}, \ldots, \int_{L_{r}} \emptyset\left(s_{1}, \ldots ., s_{r}\right) \prod_{i=1}^{r} \emptyset_{i}\left(s_{i}\right) z_{i} s_{i} \xi^{\lambda+\sigma_{1} s+\sigma_{2} r^{-} R} \\
& \quad \times \frac{\Gamma\left[1-\left(-\lambda-\sigma_{2} s\right)+\sigma_{1} s, \ldots, \sigma_{r} s\right]\left\lceil\left[1-\left(-m-R-\rho_{2} r\right)+\rho_{1} s, \ldots, \rho_{r} s\right]\right.}{(R) ! \Gamma\left[1-\left(R-\lambda-\sigma_{2} S\right)+\sigma_{1} s, \ldots, \sigma_{r} s\right]\left\lceil\left[1-\left(\vartheta-m-R-\rho_{2} r\right)+\rho_{1} s, \ldots, \rho_{r} s\right]\right.} \\
& \quad \times\left(z_{2} x^{\rho_{2}}\right)^{r}\left(z_{3} \xi^{\sigma_{2}}\right)^{s} x^{m+\rho_{1} s+\rho_{2} r+R-\vartheta} z_{1}{ }^{s}, \ldots, z_{r}{ }^{s} \mathrm{~d} s_{1}, \ldots, d s_{r}
\end{aligned}
$$

If we interpret the resulting Mellin-Barnes contour integral as an A-function of multivariable, we shall arrive (3.1).

THEOREM 2. If $\min \left\{\rho_{r}, \sigma_{r}\right\}>0,|\arg (-x / \xi)|<\pi$, Re (m) $+\rho_{r} \min \{\operatorname{Re}$ $\left.\left(\delta_{j}, \gamma_{j}\right)\right\}>-1(j=1,2, \ldots, r),\left|z_{2}(x-\xi)^{\rho_{r}}\right|<r_{1},\left|(\eta-x)^{\sigma_{r}} z_{r}\right|<r_{2}, r_{1}+r_{2}+r_{n}=1$; then

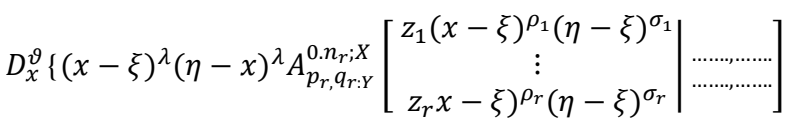

$$
\begin{aligned}
& \left.G_{1}\left(\gamma, \delta, \delta^{\prime}: Z_{2} x^{\rho_{2}},(\mathrm{x}-\xi)^{\sigma_{2}},(\eta-x)^{\sigma_{r}} Z_{3}, \ldots, \ldots, z_{r}\right)\right\} \\
& =\sum_{r, s=0}^{\infty} \frac{(\gamma)_{r+s}(\delta)_{s-r}(\delta)^{\prime}{ }_{r-s}}{(r) !(s) !}\left[\left(z_{2}(-\xi)^{\rho_{2}}\right]^{r}\left[z_{3} \eta^{\sigma_{2}}\right]^{s}(-\xi)^{m}(\eta)^{\lambda}\right. \\
& \sum_{R_{1}=0}^{\infty} \sum_{R_{2}=0}^{\infty} \frac{x^{-\vartheta}(x / \xi)^{R_{1}}(x / \eta)^{R_{2}}}{\left(R_{1}\right) !\left(R_{2}\right) !} \frac{\Gamma\left(R_{1}+R_{2}+1\right)}{\Gamma\left(R_{1}+R_{2}-\vartheta+1\right)} \\
& A_{p_{r+2,}, q_{r+2: Y}}^{0 . n_{r}+2 ; X}\left[\begin{array}{c|c}
z_{1}(-\xi)^{\rho_{1}} \eta^{\sigma_{1}} \\
\vdots & \left(-\lambda-\rho_{2} s, \rho_{1}, \ldots \ldots, \rho_{r}\right),\left(-\rho-\sigma_{2} s, \sigma_{1}, \ldots, \ldots, \sigma_{r}\right), \ldots, \ldots \ldots \\
z_{r}(-\xi)^{\rho_{r}} \eta^{\sigma_{r}} & \ldots, \ldots, \ldots\left(R_{1}-m-\rho_{2} r, \rho_{1}, \ldots, \ldots \rho_{r}\right)\left(R_{2}-\sigma_{2} s, \sigma_{1}, \ldots, \ldots, \sigma_{r}\right)
\end{array}\right] .
\end{aligned}
$$

PROOF. we first replace the A-function of several variable occurring on the left - hand side by its Mellin -Barnes type contour integral and Horn's function $G_{1}$ by its definition and changing the order of integration and differentiation, which is readily justified in view of conditions stated above and collecting the powers of $(\mathrm{x}-\xi)$ and $(\eta-$ $\mathrm{x}$ ), we get 


$$
\begin{aligned}
& \sum_{r, s=0}^{\infty} \frac{(\gamma)_{r+s}(\delta)_{s-r}(\delta)^{\prime}{ }_{r-s}}{(r) !(s) !} z_{2}{ }^{r} z_{3}{ }^{s} \frac{1}{(2 \pi \omega)^{r}} \int_{L_{1}}, \ldots ., \int_{L_{r}} \phi\left(s_{1}, \ldots . ., s_{r}\right) \prod_{i=1}^{r} \emptyset_{i}\left(s_{i}\right) z_{i} s_{i} \\
& \left.\left\{D_{x}^{v}(x-\xi)^{m+\rho_{1} s+\rho_{2} r} \times(\eta-x)^{\lambda+\sigma_{1} s+\sigma_{2} r}\right)\right\} \mathrm{d} s_{1}, \ldots, d s_{r}
\end{aligned}
$$

Now, applying well known Binomial expansion, we have

$$
\begin{aligned}
& \sum_{r, s=0}^{\infty} \frac{(\gamma)_{r+s}(\delta)_{s-r}(\delta)^{\prime}{ }_{r-s}}{(r) !(s) !} z_{2}{ }^{r} z_{3}{ }^{s} \frac{1}{(2 \pi \omega)^{r}} \int_{L_{1}}, \ldots, \int_{L_{r}} \emptyset\left(s_{1}, \ldots ., s_{r}\right) \prod_{i=1}^{r} \emptyset_{i}\left(s_{i}\right) z_{i} s_{i}(-\xi)^{m+\rho_{1} s+\rho_{2} r} \\
& \left.\times(\eta)^{\lambda+\sigma_{1} s+\sigma_{2} r} D_{x}^{v} \sum_{R_{1}=0}^{\infty}\left(\begin{array}{c}
m+\rho_{1} s+\rho_{2} r \\
R_{1}
\end{array}\right)\left(\frac{-x}{\xi}\right)^{R_{1}} \sum_{R_{2}=0}^{\infty}\left(\begin{array}{c}
\lambda+\sigma_{1} s+\sigma_{2} r \\
R_{2}
\end{array}\right)\left(\frac{-x}{\eta}\right)^{R_{2}}\right\} \\
& z_{1}{ }^{s}, \ldots, z_{r} s \mathrm{~d} s_{1}, \ldots, d s_{r}
\end{aligned}
$$

Making the use of the formula [the result Oldham and Spanier [9]], we get

$$
\begin{aligned}
& \sum_{r, s=0}^{\infty} \frac{(\gamma)_{r+s}(\delta)_{s-r}(\delta)^{\prime}{ }_{r-s}}{(r) !(s) !} z_{2}{ }^{r} z_{3}{ }^{s}(-\xi)^{m+\rho_{1} s}(\eta)^{\lambda+\sigma_{1} s} \sum_{R_{1}=0}^{\infty} \sum_{R_{2}=0}^{\infty} \frac{(-1)^{R_{1}+R_{2}}\left(\frac{x}{\xi}\right)^{R_{1}}\left(\frac{x}{\eta}\right)^{R_{2}} x^{-\vartheta}}{\left(R_{1}\right) !\left(R_{2}\right) !} \\
& \frac{\Gamma\left(R_{1}+R_{2}+1\right)}{\Gamma\left(R_{1}+R_{2}-\vartheta+1\right)} \frac{1}{(2 \pi \omega)^{r}} \int_{L_{1}}, \ldots, \int_{L_{r}} \phi\left(s_{1}, \ldots ., s_{r}\right) \prod_{i=1}^{r} \emptyset_{i}\left(s_{r}\right) z_{i}{ }^{s_{r}}(-\xi)^{\rho_{1} s}(-\eta)^{\sigma_{1} s} \\
& \quad \times \frac{\Gamma\left[1-\left(-m-\rho_{2} r\right)+\rho_{1} s, \ldots, \rho_{r} s\right]\left\lceil\left[1-\left(-\lambda-\sigma_{2} s\right)+\sigma_{1} s, \ldots, \sigma_{r} s\right]\right.}{\Gamma\left[1-\left(R_{1}-m-\rho_{2} r\right)+\rho_{1} s, \ldots, \rho_{r} s\right] \Gamma\left[1-\left(R_{2}-\sigma_{2} S\right)+\sigma_{1} s, \ldots, \sigma_{r} s\right]} \\
& z_{1}{ }^{s}, \ldots, z_{r}{ }^{s} \mathrm{~d} s_{1}, \ldots, d s_{r} .
\end{aligned}
$$

If we interpret the resulting Mellin -Barnes contour integral as an A- function of multivariable, we shall arrive (3.5).

\section{SPECIAL CASES OF (3.1) AND (3.5)}

(1) Putting $\sigma_{r} \rightarrow 0$ another four Fractional Derivative formulae corresponding to (3.1) and (3.5):

$$
\begin{aligned}
& \left.D_{x}^{\vartheta} x^{m}(x \xi)^{\lambda} A_{p_{r}, q_{r: Y}}^{0 . n_{r} ; X}\left[\begin{array}{c}
z_{1}(-\xi)^{\rho_{1}} \eta^{\sigma_{1}} \\
\vdots \\
z_{r}(-\xi)^{\rho_{r}} \eta^{\sigma_{r}}
\end{array} \ldots \ldots, \ldots, \ldots\right] G_{1}\left(\gamma, \delta, \delta^{\prime}: Z_{2} x^{\rho_{2}},(\mathrm{x}+\xi)^{\sigma_{2}} Z_{3}, \ldots, \ldots, z_{r}\right)\right\} \\
& =\sum_{r, s=0}^{\infty} \frac{(\gamma)_{r+s}(\delta)_{s-r}(\delta)^{\prime}{ }_{r-s}}{(r) !(s) !}\left(z_{2} x^{\rho_{2}}\right)^{r}\left(z_{3} \xi^{\sigma_{2}}\right)^{s} Z_{r} \xi^{\lambda} x^{m-\vartheta} \sum_{R=0}^{\infty} \frac{(x / \xi)}{(R) !}
\end{aligned}
$$

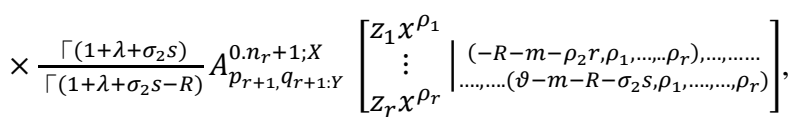




$$
\begin{aligned}
& \min \left\{\rho_{r,}\right\}>0,|\arg (x / \xi)|<\pi, \\
& \operatorname{Re}(\mathrm{m})+\rho_{r} \min \left\{\operatorname{Re}\left(\delta_{j}, \gamma_{j}\right)\right\}>-1(j=1, \ldots, r), \\
& \left|z_{r} x^{\rho_{r}}\right|<r_{1},\left|(x+\xi)^{\sigma_{r}} z_{r}\right|<r_{2}, r_{1}+r_{2}+, \ldots, r_{n}=1 \text {; }
\end{aligned}
$$

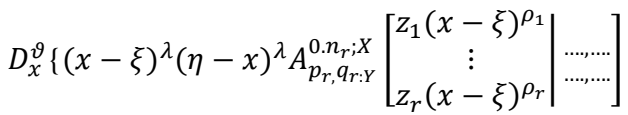

$$
\begin{aligned}
& \left.\times G_{1}\left(\gamma, \delta, \delta^{\prime}: Z_{2},(\mathrm{x}-\xi)^{\rho_{r}},(x-\eta)^{\sigma_{r}} Z_{3}, \ldots, \ldots, z_{r}\right)\right\} \\
& =\sum_{r, s=0}^{\infty} \frac{(\gamma)_{r+s}(\delta)_{s-r}(\delta)^{\prime}{ }_{r-s}}{(r) !(s) !}\left[\left(z_{2}(-\xi)^{\rho_{2}}\right]^{r}\left[z_{3} \eta^{\sigma_{2}}\right]^{s}(-\xi)^{m}(\eta)^{\lambda}\right. \\
& \sum_{R_{1}=0}^{\infty} \sum_{R_{2}=0}^{\infty} \frac{x^{-\vartheta}(x / \xi)^{R_{1}}(x / \eta)^{R_{2}}}{\left(R_{1}\right) !\left(R_{2}\right) !} \frac{\Gamma\left(R_{1}+R_{2}+1\right)}{\Gamma\left(R_{1}+R_{2}-\vartheta+1\right)} \times \frac{\Gamma\left(1+\lambda+\sigma_{r} s\right)}{\Gamma\left(1+\sigma_{r} s-R_{2}\right)} \\
& A_{p_{r+1}, q_{r+1: Y}}^{0 . n_{r}+1 ; X}\left[\begin{array}{c|c}
z_{1}(-\xi)^{\rho_{1}} & \left(-m-\rho_{2} r, \rho_{1}, \ldots, \rho_{r}\right), \ldots, \ldots \ldots \\
\vdots & \ldots, \ldots .\left(R_{1}-m-\rho_{2} r_{1}, \rho_{1}, \ldots . . . \rho_{r}\right)\left(R_{2}-\sigma_{2} s, \sigma_{1}, \ldots, \ldots, \sigma_{r}\right)
\end{array}\right], \\
& \min \left\{\rho_{r}\right\}>0,|\arg (-x / \xi)|<\pi, \\
& \operatorname{Re}(\mathrm{m})+\rho_{r} \min \left\{\operatorname{Re}\left(\delta_{j}, \gamma_{j}\right)\right\}>-1(j=1,2, \ldots, r), \\
& \left|z_{2}(x-\xi)^{\rho_{r}}\right|<r_{1},\left|(\eta-x)^{\sigma_{r}} z_{3}\right|<r_{2}, r_{1}+r_{2}+r_{n}=1 \text {; }
\end{aligned}
$$

\section{ACKNOWLEDGEMENTS}

The authors are thankful to the editor Professor Iveta Dirgová Luptáková and learned reviewer for their valuable comments towards the manuscript.

\section{REFERENCES}

[1] Berndt, B.C. and Bowman, D.C. (2000). Ramanujan's short unpublished manuscript on integrals and series related to Euler's constant. Constructive, Experimental and Nonlinear Analysis (Lumoges, 1999), 19-27, CMS Conf. Proc.27, Amer. Math. Soc., providence, RI,2000

[2] Chaurasia, V.B.L. amd Godika, A. (1999). An integral involving certain product of special functions. Bull. Calcutta Math. Soc. 91, 4, 337-342.

[3] Saxena, V.P. (2001). Formal solution of certain new pair of dual integral equations involving H-function. Proc. Nat. Acad. Sci. India Sect. A51, 366-375.

[4] Tripathi, B., Sharma, R. and Sharma, C.K. (2020). Some results of fractional integral involving I-function and general class of polynomial. International Journal of Applied and Computational Mathematics, 6:103, DOI:10.1007/s40819-020-00855-w.

[5] Gautam, B.P. and Asgar, A.S. (1980). The A-function. Revista Mathematical, Tucuman. 
[6] Gautam, B.P. and Asgar, A.S. (1986). On the multivariable A-function. Vijnana Parishas Anusandhan Patrika, 29, 4, 67-81.

[7] Ram, J. and Kumar, D. (2011). Generalized fractional integration involving Appell hypergeometric function of the product of two H-functions, Vijanana Parishad Anusandhan Patrika, 54, 3, 33-43.

[8] Srivastava, H.M. and Panda, R. (1975). Some expansion theorems and generating relations for the H- function of several complex variables, Comment. Math. Univ. St. Paul. 24, 119137.

[9] Oldham, K.B. and Spanies, J. (1974). The Fractional Calculus. Academic press, New York, London.

\section{Roshani Sharma}

Department of Mathematics, Lakshmi Nairan College of Technology, Bhopal-462021, Madhya Pradesh, India e-mail: roshnipsharma@gmail.com

\section{Bhupendra Tripathi}

Department of Mathematics, Lakshmi Nairan College of Technology, Bhopal-462021, Madhya Pradesh, India e-mail: btripathirewa@yahoo.co.in

\section{Anamika Dubey}

Department of Mathematical Science,

A.P.S. University, Rewa-486003, Madhya Pradesh, India. e-mail: anamikadubeyji1992@gmail.com 